MSC 65M06, 65M12

DOI: $10.14529 /$ jcem200404

\title{
A COUPLED UNIFIED DIFFERENCE METHOD FOR NUMERICAL SOLUTION OF THE INITIAL BOUNDARY VALUE FOURTH ORDER PARABOLIC PROBLEMS
}

P. K. Pandey, Dyal Singh College (University of Delhi), Lodhi Road New Delhi, India, pramod_10p@hotmail.com

In this article we proposed a coupled unified difference method for the numerical solution for the fourth order parabolic problems. To simplify the complexity of higher order differential term, we introduced an intermediate function and hence the higher order boundary value problem is transformed into an equivalent system of reduced order boundary value problems. We have discussed the convergence of the proposed method. Numerical experiments are performed to test and approve the efficiency, accuracy of the proposed method.

Keywords: convergence analysis; coupled approach; fourth order parabolic equation; initial boundary value problem; unified difference method.

\section{Introduction}

To describe most real physical phenomena in engineering and natural science a partial differential equations and corresponding boundary value problems has very significant and important role. The effect of surface tension and capillarity on thin film, thermal diffusion on the surface of a solid, the dynamics of particle in phase transition is well described by the application of fourth order parabolic partial differential equations and corresponding BVPs. A literary application of parabolic PDEs are there in the modelling and studies of physical phenomena such as the thermal diffusion on surface [1,2], theory of thin film [3-5] and dynamics of phase transition [6-8].

In this article we consider following fourth order parabolic BVPs,

$$
\frac{\partial u}{\partial t}=-\frac{\partial^{4} u}{\partial x^{4}}+\alpha \frac{\partial^{3} u}{\partial x^{3}}+f\left(x, t, u, \frac{\partial u}{\partial x}, \frac{\partial^{2} u}{\partial x^{2}}, \frac{\partial^{3} u}{\partial x^{3}}\right), \quad a<x<b, \quad 0 \leq t<T,
$$

subject to initial-boundary conditions

$$
\begin{gathered}
u(x, 0)=u_{0}(x), \quad u(a, t)=b_{0}(t), \quad u(b, t)=b_{1}(t), \\
\frac{\partial^{2} u(a, t)}{\partial x^{2}}=d_{0}(t) \quad \text { and } \quad \frac{\partial^{2} u(b, t)}{\partial x^{2}}=d_{1}(t) .
\end{gathered}
$$

where $u_{0}(x), b_{0}(t), b_{1}(t), d_{0}(t)$ and $d_{1}(t)$ are real constant or real function and $f\left(x, t, \frac{\partial u}{\partial x}, \frac{\partial^{2} u}{\partial x^{2}}, \frac{\partial^{3} u}{\partial x^{3}}\right)$ is continuous in $[a, b], t \geq 0$.

The solution of the partial differential equations and corresponding boundary value problems is an extremely important and interesting area of research. Generally, we face problem in finding analytically closed form of the solution of non linear problem even when 
it exists. So we will not consider any specific condition on forcing function in problem (1) to ensure the existence and uniqueness of solution of the problem. We have assumed that considered problem (1) is well posed.

An accurate modelling of physical phenomena required to solve the generally involving more complexity and nonlinearity in PDEs that govern the physical phenomena. Because of the inability in finding a closed form analytical solution, numerical techniques for finding approximate solutions to these PDEs are sought. Over a last few decades, a literary work on numerical solution technique for accurate numerical solution of the PDEs in particular fourth order parabolic PDEs are reported in the literature on the numerical analysis. Some of these existing numerical techniques for solving problems (1) employ Finite Difference Method [9, 10], Spline Method [11], Differential transformation Method [12], Homotopy Perturbation Method [13] and references therein.

To simplify complexity and nonlinearity approximations is made to reduce the governing PDEs to system of differential equations / algebraic equations and hence reduced problem solved. A continuous problem transformed into a discrete problem by application of Taylor's series approximations. The difference equations are accurate and efficient if the approximate solution of the problem is close to the exact solution of the problem and these difference equations are more accurate than those obtained by application of Taylor's series approximations. The emphasis in this article will be on the development of an accurate and efficient, unified difference method for the approximate numerical solution of the problem (1) by the application of Taylor's series expansion and other techniques.

The outline of this article is as follows. In Section 2, we replace problem (1) in system of boundary value problems and develop a unified difference method to solve the resulting system of BVPs. In Section 3, we prove the convergence property of the proposed method. In section 4, the numerical results so produced applying the method of the article on two model problems are presented. Finally a discussion and conclusion on the performance of the proposed method are presented in Section 5.

\section{Unified Difference Method}

In this section we propose unified difference method for the numerical solution of the problem (1). Let us introduce an intermediate variable $v(x, t)$ such that

$$
\frac{\partial^{2} u}{\partial x^{2}}=v(x, t)
$$

and the boundary conditions are

$$
u(a, t)=b_{0}(t), \quad u(b, t)=b_{1}(t)
$$

This intermediate variable enable us to transform problem (1) into following an equivalent problem

$$
\frac{\partial u}{\partial t}=-\frac{\partial^{2} v}{\partial x^{2}}+\alpha \frac{\partial v}{\partial x}+f\left(x, t, u, \frac{\partial u}{\partial x}, v, \frac{\partial v}{\partial x}\right),
$$

subject to transformed initial boundary conditions are

$$
u(x, 0)=u_{0}(x), \quad v(a, t)=d_{0}(t) \quad \text { and } \quad v(b, t)=d_{1}(t) .
$$


Thus, problem (1) is reduced into an equivalent coupled system of differential equations (2-3) subject transformed initial and boundary conditions. Also the initial condition $u(x, 0)=u_{0}(x)$, enable us to compute the values of $\frac{\partial^{r} u}{\partial x^{r}}, r=1,2 .$. at $t=0$. So we have the value of intermediate variable $v(x, t)$ at $t=0$.

We substitute rectangular domain $[a, b] \times[0, T]$ by a discrete set of mesh points and we wish to determine the numerical solution of the problem (1) at these discrete mesh points. Let $h=(b-a) /(N+1)$ and $k$ be the step size respectively in the $x$ and $t$ directions of the Cartesian coordinate system parallel to coordinate axes. Thus we have generated mesh points $\left(x_{i}, t_{j}\right) ; x_{i}=a+i h, i=0,1, \ldots, N+1$ and $t_{j}=j k, j=0,1, . ., M$. Let us denote the numerical approximation of $u(x, t)$ at mesh point $\left(x_{i}, t_{j}\right)$ by $u_{i, j}$ for $i=0,1, \ldots, N+1$ and $j=0,1, . ., M$. Let us denote approximation of the theoretical value of the forcing function $f\left(x, t, u(x), \frac{\partial u}{\partial x}, v(x), \frac{\partial v}{\partial x}\right)$ at node $\left(x_{i}, y_{j}\right)$ as $f_{i . j}, \quad i=0,1,2, \ldots . ., N+1, \quad j=0,1, . ., M$. Thus, using these finite difference, the problem (2-3) reduced to the following discrete problem at node $\left(x_{i}, y_{j}\right)$,

$$
u_{i, j}^{\prime \prime}=v_{i, j}, \quad \dot{u}_{i, j}=v_{i, j}^{\prime \prime}-\alpha v_{i, j}^{\prime}+f_{i, j}, \quad i=0,1, \cdots, N+1, \quad j=0,1, \cdots, M,
$$

where $\dot{u}=\frac{\partial u}{\partial t}, v^{\prime \prime}=\frac{\partial^{2} u}{\partial x^{2}}$ and subject to the initial boundary conditions

$$
u_{i, 0}=u_{0}\left(x_{i}\right), \quad u_{0, j}=b_{0}\left(t_{j}\right), \quad u_{N+1, j}=b_{1}\left(t_{j}\right), \quad v_{0, j}=d_{0}\left(t_{j}\right) \quad \text { and } \quad v_{N+1, j}=d_{1}\left(t_{j}\right) .
$$

Let define following approximations,

$$
\begin{aligned}
& \bar{v}_{i, j}^{\prime}=\frac{v_{i+1, j}-v_{i-1, j}}{2 h} \bar{u}_{i, j}^{\prime}=\frac{u_{i+1, j}-u_{i-1, j}}{2 h} \\
& \text { and } \bar{f}_{i, j}=f\left(x_{i}, t_{j}, u_{i, j}, \bar{u}_{i, j}^{\prime}, v_{i, j}, \bar{v}_{i, j}^{\prime}\right) .
\end{aligned}
$$

Hence, following the ideas in [14,15], we propose following unified difference method for the numerical solution of the (1),

$$
\begin{gathered}
u_{i+1, j}-2 u_{i, j}+u_{i-1, j}=h^{2} v_{i, j} \\
v_{i+1, j}-(1+\exp (\alpha h)) v_{i, j}+\exp (\alpha h) v_{i-1, j}=\frac{h^{2}(1+\exp (\alpha h))}{2}\left(\bar{f}_{i, j}-\dot{u}_{i, j}\right) .
\end{gathered}
$$

Thus we have obtained the system of equations (6) at each interior mesh point $\left(x_{i}, t_{j}\right): i=1,2, \cdots, N$ and $j=1,2, \cdots$ of the discrete domain. Thus we have an implicit finite difference methods which can be written in block tri-diagonal matrix form and can be solved by iterative methods. The solution of the system of equations (6) is the solution of the problem (1) in the discrete domain.

\section{Convergence Analysis}

In this section we will discuss convergence of the proposed method (6). Let us consider following test equation,

$$
\frac{\partial u}{\partial t}=-\frac{\partial^{2} v}{\partial x^{2}}+\alpha \frac{\partial v}{\partial x}+f\left(x, t, u, \frac{\partial u}{\partial x}, v, \frac{\partial v}{\partial x}\right) .
$$


subject to transformed initial boundary conditions are

$$
u(x, 0)=u_{0}(x), \quad v(a, t)=d_{0}(t) \quad \text { and } \quad v(b, t)=d_{1}(t),
$$

subject to the boundary conditions $y(a)=\beta, y(b)=\gamma, y^{\prime \prime}(a)=\beta_{0}$ and $y^{\prime \prime}(b)=\gamma_{0}$.

Let $U_{i, j}, V_{i, j}$ and $F_{i, j}$ are respectively the exact value of solution $u(x, t)$ of the problem (7), intermediate function $v(x, t)$ and forcing function $f\left(x, t, u, \frac{\partial u}{\partial x}, v, \frac{\partial v}{\partial x}\right)$ at the mesh point $\left(x_{i}, t_{j}\right)$. Thus we have,

$$
F_{i, j}=f\left(x_{i}, t_{j}, U_{i, j}, U_{i, j}^{\prime}, V_{i, j}, V_{i, j}^{\prime}\right) \quad \text { and } \quad f_{i, j}=f\left(x_{i}, t_{j}, u_{i, j}, u_{i, j}^{\prime}, v_{i, j}, v_{i, j}^{\prime}\right) .
$$

So, we write difference method (6) for the numerical solution of the considered problem (7) in the following form using above defined notations,

$$
\begin{gathered}
U_{i+1, j}-2 U_{i, j}+U_{i-1, j}-h^{2} V_{i, j}=T_{1 i, j} \\
V_{i+1, j}-(1+\exp (\alpha h)) V_{i, j}+\exp (\alpha h) V_{i-1, j}+\frac{h^{2}(1+\exp (\alpha h))}{2} \dot{U}_{i, j} \\
=\frac{h^{2}(1+\exp (\alpha h))}{2} \bar{F}_{i, j}+T_{2 i, j} .
\end{gathered}
$$

and

$$
\begin{gathered}
u_{i+1, j}-2 u_{i, j}+u_{i-1, j}-h^{2} v_{i, j}=0 \\
v_{i+1, j}-(1+\exp (\alpha h)) v_{i, j}+\exp (\alpha h) v_{i-1, j}+\frac{h^{2}(1+\exp (\alpha h))}{2} \dot{u}_{i, j} \\
=\frac{h^{2}(1+\exp (\alpha h))}{2} \bar{f}_{i, j}
\end{gathered}
$$

where $T_{1 i, j}$ and $T_{2 i, j}$ are local truncation errors at mesh point $\left(x_{i}, t_{j}\right)$. It is easy to prove that approximations (5) provides $O\left(h^{2}\right)$ for the forcing function $f_{i, j}$, i.e.

$$
\bar{f}_{i, j}=f_{i, j}+O\left(h^{2}\right)
$$

and similarly we have,

$$
\bar{F}_{i, j}=F_{i, j}+O\left(h^{2}\right)
$$

Let us define local truncation error in approximate values of solution $u(x, t)$ and intermediate function $v(x, t)$, i.e.

$$
\epsilon_{i, j}=U_{i, j}-u_{i, j} \quad \text { and } \quad \delta_{i, j}=V_{i, j}-v_{i, j}, \quad i=1,2, \cdots, N .
$$

Subtract (9) from (8) and using above approximations we have obtained following,

$$
\begin{gathered}
\epsilon_{i+1, j}-2 \epsilon_{i, j}+\epsilon_{i-1, j}-h^{2} \delta_{i, j}=T_{1 i, j} \\
\delta_{i+1, j}-(1+\exp (\alpha h)) \delta_{i, j}+\exp (\alpha h) \delta_{i-1, j}+\frac{h^{2}}{2}(1+\exp (\alpha h))\left(\dot{U}_{i, j}-\dot{u}_{i, j}\right) \\
=\frac{h^{2}}{2}(1+\exp (\alpha h))\left(F_{i, j}-f_{i, j}\right)+T_{2 i, j},
\end{gathered}
$$


where

$$
\begin{gathered}
T_{1 i, j}=\frac{h^{4}}{12}\left(\frac{\partial^{4} u}{\partial x^{4}}\right)_{i, j} \\
T_{2 i, j}=\frac{h^{4}}{12}\left(\frac{\partial^{4} v}{\partial x^{4}}-2 \alpha \frac{\partial^{3} v}{\partial x^{3}}+3 \alpha^{2} \frac{\partial^{2} v}{\partial x^{2}}-3 \alpha^{3} \frac{\partial v}{\partial x}\right)_{i, j}, \quad i=1,2, \cdots, N, \quad j=1,2, \cdots, M .
\end{gathered}
$$

Let we linearize $F_{i, j}$ and write as

$$
F_{i, j}-f_{i, j}=\left(U_{i, j}-u_{i, j}\right) G_{i, j}+\left(U_{i, j}^{\prime}-u_{i, j}^{\prime}\right) \dot{G}_{i, j}+\left(V_{i, j}-v_{i, j}\right) H_{i, j}+\left(V_{i, j}^{\prime}-v_{i, j}^{\prime}\right) \dot{H}_{i, j}
$$

where

$$
G_{i, j}=\left(\frac{\partial f}{\partial u}\right)_{i, j}, \quad \dot{G}_{i, j}=\left(\frac{\partial f}{\partial u^{\prime}}\right)_{i, j}, \quad H_{i, j}=\left(\frac{\partial f}{\partial v}\right)_{i, j} \quad \text { and } \quad \dot{H}_{i, j}=\left(\frac{\partial f}{\partial v^{\prime}}\right)_{i, j} .
$$

Hence,

$$
F_{i, j}-f_{i, j}=\epsilon_{i, j} G_{i, j}+\frac{1}{2 h}\left(\epsilon_{i+1, j}-\epsilon_{i-1, j}\right) \dot{G}_{i, j}+\delta_{i, j} H_{i, j}+\frac{1}{2 h}\left(\delta_{i+1, j}-\delta_{i-1, j}\right) \dot{H}_{i, j}
$$

Substituting $F_{i, j}-f_{i, j}$ from (11) in (10) and approximating $\dot{U}_{i, j}-\dot{u}_{i, j}$ by the finite difference, we have obtained following error equations in matrix form after incorporating boundary conditions,

$$
(\mathbf{J}+\mathbf{L}) \mathbf{E}=\mathbf{T}
$$

These matrices are,

$$
\begin{gathered}
\mathbf{J}=\left(\begin{array}{ccc}
\mathbf{A}_{1,1} & \vdots & \mathbf{A}_{1,2} \\
\cdots & \cdots & \cdots \\
\mathbf{A}_{2,1} & \vdots & \mathbf{A}_{2,2}
\end{array}\right)_{2 N \times 2 N}, \mathbf{L}=\left(\begin{array}{ccc}
\mathbf{0} & \vdots & \mathbf{0} \\
\cdots & \cdots & \cdots \\
\mathbf{L}_{2,1} & \vdots & \mathbf{L}_{2,2}
\end{array}\right)_{2 N \times 2 N} \\
\mathbf{E}=\left(\epsilon_{1, j}, \cdots, \epsilon_{N, j}, \delta_{1, j}, \cdots, \delta_{N, j}\right)^{T} \\
\mathbf{T}=\left(T_{11, j}, \cdots, T_{1 N, j}, \bar{T}_{21, j}, \cdots, \bar{T}_{2 N, j}\right)^{T}
\end{gathered}
$$

Further we define each block matrix,

$$
\begin{aligned}
& \mathbf{A}_{1,1}=\left(\begin{array}{cccc}
-2 & 1 & & 0 \\
1 & -2 & 1 & \\
& \ddots & \ddots & \\
0 & & 1 & -2
\end{array}\right)_{N \times N}, \mathbf{A}_{1,2}=-h^{2} \mathbf{I}_{N} \\
& \mathbf{A}_{2,1}=\frac{h^{2}(1+\exp (\alpha h))}{2 k} \mathbf{I}_{N}, \\
& \mathbf{A}_{2,2}=\left(\begin{array}{cccc}
-(1+\exp (\alpha h)) & 1 & & 0 \\
\exp (\alpha h) & -(1+\exp (\alpha h)) & 1 & \\
0 & \ddots & \ddots & \\
& & \exp (\alpha h) & -(1+\exp (\alpha h))
\end{array}\right)_{N \times N},
\end{aligned}
$$




$$
\begin{gathered}
\mathbf{L}_{2,1}=-\frac{h^{2}(1+\exp (\alpha h))}{2}\left(\begin{array}{cccc}
G_{1, j} & \frac{1}{2 h} \dot{G}_{1, j} & & 0 \\
-\frac{1}{2 h} \dot{G}_{2, j} & G_{2, j} & \frac{1}{2 h} \dot{G}_{2, j} & \\
& \ddots & \ddots & \\
0 & & -\frac{1}{2 h} \dot{G}_{N, j} & G_{N, j}
\end{array}\right)_{N \times N}, \\
\mathbf{L}_{2,2}=-\frac{h^{2}(1+\exp (\alpha h))}{2}\left(\begin{array}{cccc}
H_{1, j} & \frac{1}{2 h} \dot{H}_{1, j} & & 0 \\
-\frac{1}{2 h} \dot{H}_{2, j} & H_{2, j} & \frac{1}{2 h} \dot{H}_{2, j} & \\
& \ddots & \ddots & \\
0 & & -\frac{1}{2 h} \dot{H}_{N, j} & H_{N, j}
\end{array}\right)_{N \times N}
\end{gathered}
$$

and

$$
\bar{T}_{2 i, j}=T_{2 i, j}-\frac{k h^{2}(1+\exp (h \alpha))}{4}\left(\frac{\partial^{2} u}{\partial t^{2}}\right)_{i, j}
$$

Let

$$
G=\max _{1 \leq i, j \leq N}\left|G_{i, j}\right|, \quad \dot{G}=\max _{1 \leq i, j \leq N}\left|\dot{G}_{i, j}\right|, H=\max _{1 \leq i, j \leq N}\left|H_{i, j}\right|, \quad \dot{H}=\max _{1 \leq i, j \leq N}\left|\dot{H}_{i, j}\right| .
$$

So it is easy to calculate $\left\|\mathbf{L}_{2,1}\right\|$ and $\left\|\mathbf{L}_{2,2}\right\|$. Matrix $\mathbf{A}_{1,1}$ is invertible [16]. We determined $\mathbf{A}_{2,2}^{-1}=\left(a_{l, m}\right)$ explicitly, where

$$
a_{l, m}= \begin{cases}\frac{(1-\exp (l h \alpha))(\exp (-N h \alpha)-\exp ((1-m) h \alpha))}{(\exp (h \alpha)-1)(\exp (-N h \alpha)-\exp (h \alpha))}, & l \leq m \\ \frac{(1-\exp (m h \alpha))(\exp (-(N-l) h \alpha)-\exp (h \alpha))}{(\exp (h \alpha)-1)(\exp (-N h \alpha)-\exp (h \alpha))}, & l \geq m\end{cases}
$$

Let us define

$$
\bar{\Delta}=\left\|\mathbf{A}_{1,2} \mathbf{A}_{2,2}^{-1}\right\| \quad \text { and } \quad \underline{\Delta}=\left\|\mathbf{A}_{2,1} \mathbf{A}_{1,1}^{-1}\right\|
$$

and assume $\bar{\Delta} \underline{\Delta}<1$ then $\mathbf{J}$ is invertible [17]. Moreover,

$$
\left\|\mathbf{J}^{-1}\right\| \leq \frac{\max \left(\left\|\mathbf{A}_{1,1}^{-1}\right\|, \| \mathbf{A}_{2,2}^{-1}||\right)(\bar{\Delta}+1)(\underline{\Delta}+1)}{1-\underline{\Delta} \bar{\Delta}} .
$$

Let us assume $\left\|\mathbf{J}^{-1} \mathbf{L}\right\|<1$ then by [18],

$$
\left\|(\mathbf{J}+\mathbf{L})^{-1}\right\| \leq \frac{\left\|\mathbf{J}^{-1}\right\|}{1-\left\|\mathbf{J}^{-1} \mathbf{L}\right\|} .
$$

Hence using (13) in (14), we have

$$
\left\|(\mathbf{J}+\mathbf{L})^{-1}\right\| \leq \frac{\max \left(\left\|\mathbf{A}_{1,1}^{-1}\right\|, \| \mathbf{A}_{2,2}^{-1}||\right)(\bar{\Delta}+1)(\underline{\Delta}+1)}{\left(1-\left\|\mathbf{J}^{-1} \mathbf{L}\right\|\right)(1-\underline{\Delta} \bar{\Delta})} .
$$

Let

$$
M=\max \left\{\frac{h^{2}}{3}\left(\frac{\partial^{4} v}{\partial x^{4}}-2 \alpha \frac{\partial^{3} v}{\partial x^{3}}+3 \alpha^{2} \frac{\partial^{2} v}{\partial x^{2}}-3 \alpha^{3} \frac{\partial v}{\partial x}\right)-k(1+\exp (h \alpha)) \frac{\partial^{2} u}{\partial t^{2}}, \frac{h^{2}}{3} \frac{\partial^{4} u}{\partial x^{4}}\right\}
$$


for all $(x, t)$ in $[a, b] \times[0, T]$. Thus

$$
\|\mathbf{T}\| \leq \frac{h^{2}}{4} M
$$

From (12), (15) and (16), we obtained

$$
\|\mathbf{E}\| \leq \frac{h^{2}}{4} \frac{\max \left(\left\|\mathbf{A}_{1,1}^{-1}\right\|, \| \mathbf{A}_{2,2}^{-1}||\right)(\bar{\Delta}+1)(\underline{\Delta}+1)}{\left(1-\left\|\mathbf{J}^{-1} \mathbf{L}\right\|\right)(1-\underline{\Delta})} M .
$$

Thus from (17) we find $\|\mathbf{E}\|$ is bounded and $\|\mathbf{E}\|$ tends to zero as $h \longrightarrow 0$. So we conclude that proposed unified difference method (6) converge and the order of the convergence is at least $O\left(k+h^{2}\right)$.

If we replace $\dot{u}_{i, j}$ by second order differences $\frac{u_{i, j+1}-u_{i, j-1}}{2 k}$ in (6) then the method (6) has an improved order $O\left(k^{2}+h^{2}\right)$. However, in computations we replace $u_{i, j}$ by the mean of the values $u_{i, j+1}$ and $u_{i, j-1}$. The convergence of the improved method will be easily established by changing block matrix $\mathbf{A}_{2,1}$ and local truncation error $\bar{T}_{2 i, j}$.

\section{Numerical Results}

In this section, we have tested the computational efficiency of our proposed method (6) by considering two model problems. In each model problem, we took a uniform step size $h$ and $k$ respectively space and time direction. We have shown the maximum absolute error $E M U$ in the solution $u(x, t)$ of the problem (1) and the maximum absolute error $E M V$ in the intermediate function i.e. second partial derivative of solution, i.e. $\frac{\partial^{2} u(x, t)}{\partial x^{2}}=v(x, t)$ of the problems (1) for different values of $N$ and $M$ at $t=.5, .6, \cdots, 1,2$. For computation purpose we have used following formulas,

$$
\begin{aligned}
& E M U=\max _{1 \leq i \leq N}\left|U\left(x_{i}, t_{M}\right)-y_{i, M}\right|, \\
& E M V=\max _{1 \leq i \leq N}\left|V\left(x_{i}, t_{M}\right)-v_{i, M}\right| .
\end{aligned}
$$

We have used Gausss-Seidel and Newton-Raphson iteration method to solve respectively the system of linear and nonlinear equations arised from equation (6). All computations were performed on a Windows 7 Home Basic operating system in the GNU FORTRAN environment version 99 compiler (2.95 of gcc) on Intel Core i3-2330M, 2.20 Ghz PC. The iteration is continued until either the maximum difference between two successive iterates is less than $10^{-10}$ or the number of iteration reached $2 \times 10^{4}$.

Problem 1. The model non-linear problem given by

$$
\frac{\partial u}{\partial t}=-\frac{\partial^{4} u}{\partial x^{4}}-u \frac{\partial u}{\partial x}+\frac{\partial^{2} u}{\partial x^{2}}+f(x, t), \quad 0<x<1, \quad t \geq 0
$$

subject to initial and boundary conditions

$$
\begin{gathered}
u(x, 0)=\exp (\alpha x), \quad u(0, t)=\exp (t), \quad u(1, t)=\exp (\alpha+t), \\
\frac{\partial^{2} u(0, t)}{\partial x^{2}}=\alpha^{2} \exp (t) \quad \text { and } \quad \frac{\partial^{2} u(1, t)}{\partial x^{2}}=\alpha^{2} \exp (\alpha+t),
\end{gathered}
$$


where $f(x, t)$ is calculated so that the analytical solution of the problem is $u(x, t)=\exp (\alpha x+t)$. The $E M U$ and $E M V$ computed by method (6) for different values of $N, \alpha$ and $t$ are presented in Table 1 - Table 2 .

Problem 2. The model non-linear problem given by

$$
\frac{\partial u}{\partial t}=-\nu \frac{\partial^{4} u}{\partial x^{4}}+\frac{\partial^{2} u}{\partial x^{2}}+u\left(1-u^{2}\right)+f(x, t), \quad 0<x<1, \quad t \geq 0,
$$

subject to initial and boundary conditions

$$
\begin{gathered}
u(x, 0)=\exp (A x), \quad u(0, t)=\exp (-t), \quad u(1, t)=\exp (A-t), \\
\frac{\partial^{2} u(0, t)}{\partial x^{2}}=A^{2} \exp (-t) \quad \text { and } \quad \frac{\partial^{2} u(1, t)}{\partial x^{2}}=A^{2} \exp (A-t),
\end{gathered}
$$

where $f(x, t)$ is calculated so that the analytical solution of the problem is $u(x, t)=\exp (A x-t)$ and $A=\frac{\alpha}{\nu}$. The EMU and EMV computed by method (6) for different values of $N, \nu, \alpha$ and $t$ are presented in Table 3 - Table 4 .

\begin{tabular}{|c|c|c|c|c|c|}
\hline \multirow[b]{2}{*}{$\alpha$} & \multirow[b]{2}{*}{$\mathrm{t}$} & \multirow[b]{2}{*}{ M } & \multirow[b]{2}{*}{$\mathrm{N}+1$} & \multicolumn{2}{|c|}{ Maximum absolute error } \\
\hline & & & & $E M U$ & $E M V$ \\
\hline \multirow{5}{*}{1.75} & \multirow{5}{*}{1} & \multirow{5}{*}{75} & 16 & $.64945221(-3)$ & $.32990754(-1)$ \\
\hline & & & 32 & $.29373169(-3)$ & $.43669939(-2)$ \\
\hline & & & 64 & $.18024445(-3)$ & $.15412271(-2)$ \\
\hline & & & 128 & $.22888184(-4)$ & $.50322413(-2)$ \\
\hline & & & 256 & $.95367432(-6)$ & $.15139580(-4)$ \\
\hline \multirow{5}{*}{1.50} & \multirow{5}{*}{1} & \multirow{5}{*}{150} & 16 & $.11205673(-3)$ & $.13374329(-1)$ \\
\hline & & & 32 & $.16164780(-3)$ & $.15201569(-2)$ \\
\hline & & & 64 & $.54836273(-4)$ & $.95522404(-3)$ \\
\hline & & & 128 & $.95367432(-6)$ & $.27978420(-3)$ \\
\hline & & & 256 & $.19073486(-5)$ & $.39339066(-5)$ \\
\hline \multirow{5}{*}{1.25} & \multirow{5}{*}{1} & \multirow{5}{*}{300} & 16 & $.63896179(-4)$ & $.49315393(-2)$ \\
\hline & & & 32 & $68187714(-4)$ & $.59708953(-3)$ \\
\hline & & & 64 & $.95367432(-6)$ & $.26038289(-3)$ \\
\hline & & & 128 & $.95367432(-6)$ & $.16329288(-2)$ \\
\hline & & & 256 & $.95367432(-6)$ & $.23841858(-5)$ \\
\hline
\end{tabular}

\section{Table 1}

Maximum absolute error (Problem 1)

We observe in Table 2 and Table 4 respectively, for the numerical results of example 1 and example 2, $M A U$ does not depend on parameters $\alpha$ and $\nu$. The maximum absolute error at $t \in[0.5,2]$ decreases for different decreasing values of step size $h$ and $k$ in space 
Table 2

\begin{tabular}{|c|c|c|c|c|c|c|}
\hline & & & & & & \\
\hline \multirow[b]{2}{*}{$\mathrm{N}+1$} & \multirow[b]{2}{*}{$\alpha$} & \multirow[b]{2}{*}{$M$} & \multirow[b]{2}{*}{ Error } & \multicolumn{3}{|c|}{ Maximum absolute error } \\
\hline & & & & $t=0.5$ & $t=0.6$ & $t=0.7$ \\
\hline \multirow{4}{*}{16} & \multirow[t]{2}{*}{1.75} & \multirow[t]{2}{*}{75} & $E M U$ & $.18978119(-3)$ & $.18024445(-3)$ & $.19812584(-3)$ \\
\hline & & & $E M V$ & $.16171932(-1)$ & $.18483500(-1)$ & $.21257430(-1)$ \\
\hline & \multirow[t]{2}{*}{1.50} & \multirow[t]{2}{*}{150} & $E M U$ & $.15735626(-3)$ & $.15211105(-3)$ & $.14209747(-3)$ \\
\hline & & & $E M V$ & $.63347816(-2)$ & $.72772503(-2)$ & $.84204674(-2)$ \\
\hline \multirow{4}{*}{32} & \multirow[t]{2}{*}{1.75} & \multirow[t]{2}{*}{75} & $E M U$ & $.15592575(-3)$ & .1811981 & $.21505356(-3)$ \\
\hline & & & $E M V$ & $.28576851(-2)$ & $.30121803(-2)$ & $.32749474(-2)$ \\
\hline & \multirow[t]{2}{*}{1.50} & \multirow[t]{2}{*}{150} & $E M U$ & $.97274780(-4)$ & $.10776520(-3)$ & $.12159348(-3)$ \\
\hline & & & $E M V$ & $.10313988(-2)$ & $.10564327(-2)$ & $.12412071(-2)$ \\
\hline \multirow{4}{*}{64} & \multirow[t]{2}{*}{1.75} & \multirow[t]{2}{*}{75} & $E M U$ & $.13828278(-4)$ & $.18596649(-4)$ & $.51498413(-4)$ \\
\hline & & & $E M V$ & $.79214573(-4)$ & $.26896596(-4)$ & $.32812357(-3)$ \\
\hline & \multirow[t]{2}{*}{1.50} & \multirow[t]{2}{*}{150} & $E M U$ & $.95367432(-6)$ & $.14305115(-5)$ & $.54836273(-5)$ \\
\hline & & & $E M V$ & $.70035458(-4)$ & $.18882751(-3)$ & $.33116341(-3)$ \\
\hline \multirow{4}{*}{128} & \multirow[t]{2}{*}{1.75} & \multirow[t]{2}{*}{75} & $E M U$ & $.95367432(-6)$ & $.95367432(-6)$ & $.95367432(-6)$ \\
\hline & & & $E M V$ & $.13421476(-2)$ & $.18303394(-2)$ & $.54281950(-3)$ \\
\hline & \multirow[t]{2}{*}{1.50} & \multirow[t]{2}{*}{150} & $E M U$ & $.47683716(-6)$ & $.47683716(-6)$ & $.47683716(-6)$ \\
\hline & & & $E M V$ & $.24437904(-5)$ & $.26226044(-5)$ & $.29802322(-5)$ \\
\hline \multirow{4}{*}{256} & \multirow[t]{2}{*}{1.75} & \multirow[t]{2}{*}{75} & $E M U$ & $.95367432(-6)$ & $.95367432(-6)$ & $.95367432(-6)$ \\
\hline & & & $E M V$ & $.41037798(-4)$ & $.24318695(-4)$ & $.16659498(-4)$ \\
\hline & \multirow[t]{2}{*}{1.50} & \multirow[t]{2}{*}{150} & $E M U$ & $.47683716(-6)$ & $.47683716(-6)$ & $.47683716(-6)$ \\
\hline & & & $E M V$ & $.24437904(-5)$ & $.26226044(-5)$ & $.29802322(-5)$ \\
\hline
\end{tabular}

and time direction respectively. The order of accuracy in numerical solution of example 1 is approximately equal to that of theoretical estimation. However, in example 2, method converges and order of accuracy does not approve the estimated order.

\section{Conclusion}

We have described and developed coupled approach a unified difference method for numerical solution of fourth order parabolic boundary value problem. Coupled approach has been used for transforming the problem into a system of differential equations. Finally a system of differential equations which is a continuous problem transformed into a system of algebraic equations which is a discrete problem using finite differences. The proposed unified difference method use one function value at mesh point $\left(x_{i}, t_{j}\right)$ and two other mesh points $\left(x_{i \pm 1}, t_{j}\right)$. We have obtained a system of linear equations if forcing function is linear otherwise system of nonlinear equations. The proposed method is convergent 
Table 3

\begin{tabular}{|c|c|c|c|c|c|c|}
\hline \multirow[b]{2}{*}{$\nu$} & \multirow[b]{2}{*}{$\alpha$} & \multirow[b]{2}{*}{$\mathrm{t}$} & \multirow[b]{2}{*}{ M } & \multirow[b]{2}{*}{$\mathrm{N}+1$} & \multicolumn{2}{|c|}{ Maximum absolute error } \\
\hline & & & & & $E M U$ & $E M V$ \\
\hline & \multirow{12}{*}{0.15} & \multirow[b]{3}{*}{0.5} & \multirow[b]{3}{*}{100} & 16 & $.31888485(-3)$ & $.30468777(-3)$ \\
\hline & & & & 32 & $.28967857(-4)$ & $.31262264(-3)$ \\
\hline & & & & 64 & $.13828278(-4)$ & $.25777891(-3)$ \\
\hline & & \multirow[b]{3}{*}{1.0} & \multirow[b]{3}{*}{200} & 16 & $.19758940(-4)$ & $.19163266(-3)$ \\
\hline & & & & 32 & $.18507242(-4)$ & $.18445961(-3)$ \\
\hline & & & & 64 & $.12338161(-4)$ & $.18784218(-3)$ \\
\hline & & \multirow[b]{3}{*}{1.5} & \multirow[b]{3}{*}{400} & 16 & $.90897083(-5)$ & $.86029992(-4)$ \\
\hline & & & & 32 & $.78380108(-5)$ & $.84420666(-4)$ \\
\hline & & & & 64 & $.33974648(-5)$ & $.74945390(-4)$ \\
\hline & & \multirow[b]{3}{*}{2.0} & \multirow[b]{3}{*}{600} & 16 & $.49173832(-5)$ & $.47869049(-4)$ \\
\hline & & & & 32 & $.42617321(-5)$ & $.42879023(-4)$ \\
\hline & & & & 64 & $.14156103(-5)$ & $.47207810(-4$ \\
\hline
\end{tabular}

Table 4

Maximum absolute error (Problem 2)

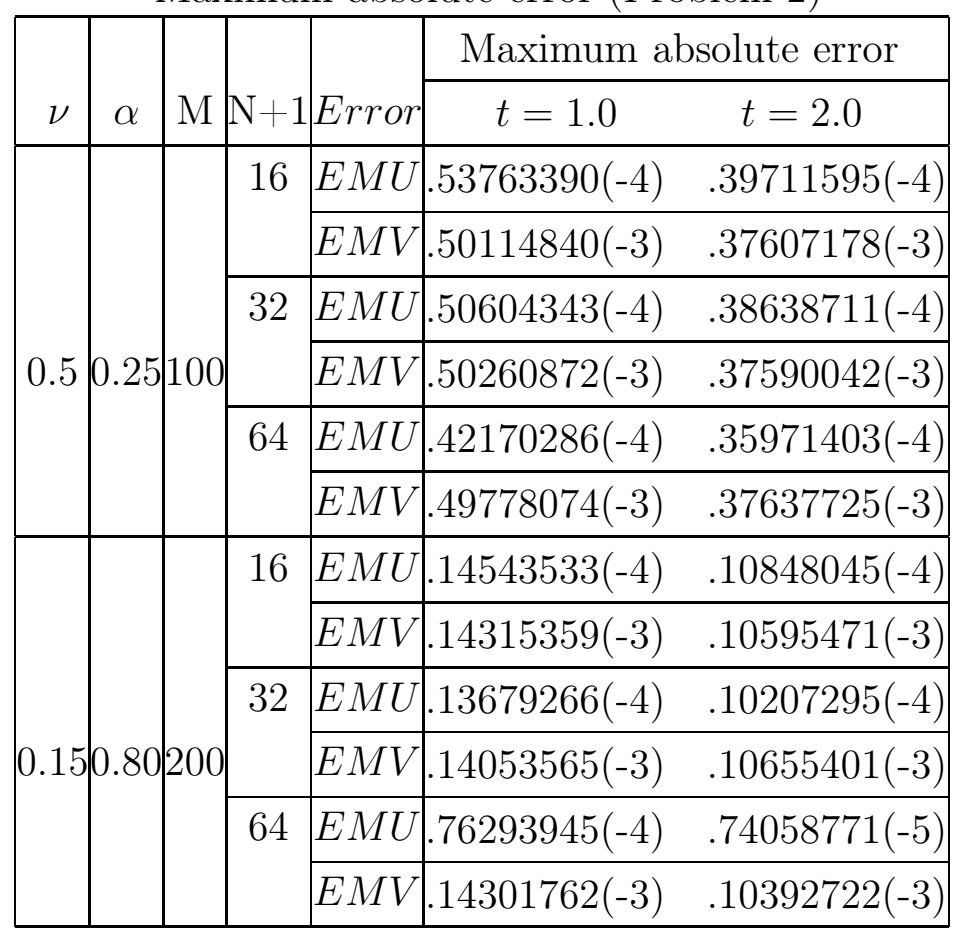

and computationally efficient. The idea presented in this article leads to the possibility to develop unified difference methods for other boundary value problems and improve the order of accuracy. Works in these directions are in progress. 


\section{References}

1. Mullins W. Theory of Thermal Grooving. Journal of Applied Physics, 1957, vol. 28, no. 3, pp. 333-339. DOI: 10.1063/1.1722742

2. Tritscher P. An Integrable Fourth-Order Nonlinear Evolution Equation Applied to Surface Redistribution due to Capillarity. Journal of the Australian Mathematical Society, Ser. B, 1997, vol. 38, no. 4, pp. 518-541. DOI: 10.1017/S0334270000000849

3. Myers T. G. Thin Films with High Surface Tension. SIAM Review, 1998, vol. 40, no. 3, pp. 441-462. DOI: 10.1137/S003614459529284X

4. Kraus H. Thin Elastic Shells. New York, John Wiley \& Sons Inc, 1967.

5. Schwartz L. W., Roy R. V. Theoretical and Numerical Results for Spin Coating of Viscous Liquids. Physics of Fluids, 2004, vol. 16, no. 3, pp. 569-584. DOI: $10.1063 / 1.1637353$

6. Allen S. M., Cahn J. W. A Microscopic Theory for Antiphase Boundary Motion and its Application to Antiphase Domain Coarsening. Acta Metall, 1979, vol. 27, pp. 1085-1095.

7. Bernoff A. J., Bertozzi A. L. Singularities in a Modified Kuramoto - Sivashinsky Equation Describing Interface Motion for Phase Transition. Physica D, 1995, vol. 85, pp. 375-404. DOI: 10.1016/0167-2789(95)00054-8

8. Rubinstein J., Sternberg P., Keller J. B. Fast Reaction, Slow Diffusion and Curve Shortening. SIAM Journal on Applied Mathematics, 1989, vol. 49, no. 1, pp. 116-133. DOI: $10.1137 / 0149007$

9. Khaliqa A. Q. M., Twizell E. H. A Family of Second Order Methods for Variable Coefficient Fourth Order Parabolic Partial Differential Equations. International Journal of Computer Mathematics, 1987, vol. 23, no. 1, pp. 63-76. DOI: $10.1080 / 00207168708803608$

10. Mohanty R. K., Evans D. J. The Numerical Solution of Fourth Order Mildly QuasiLinear Parabolic Initial Boundary Value Problem of Second Kind. International Journal of Computer Mathematics, 2003, vol. 80, no. 9, pp. 1147-1159. DOI: $10.1080 / 0020716031000148476$

11. Caglar H., Caglar N. Fifth-Degree B-spline Solution for a Fourth-Order Parabolic Partial Differential Equations. Applied Mathematics and Computation, 2008, vol. 201, no. 1, pp. 597-603. DOI: 10.1016/j.amc.2007.12.060

12. Soltanalizadeh B. Application of Differential Transformation Method for Solving a Fourth-Order Parabolic Partial Differential Equations. International Journal of Pure and Applied Mathematics, 2012, vol. 78, no. 3, pp. 299-308.

13. Dehghan M., Manafian J. The Solution of the Variable Coefficients Fourth-Order Parabolic Partial Differential Equations by the Homotopy Perturbation Method. Verlag der Zeitschrift fur Naturforschung, TuBbingen, 2009, vol. 64, pp. 420-430. DOI: $10.1515 /$ zna-2009-7-803

14. Lick W., Gaskins T. A Consistent and Accurate Procedure for Obtaining Difference Equations from Differential Equation. International Journal For Numerical Methods In Engineering, 1984, vol. 20, pp. 1433-1441. DOI: 10.1002/NME.1620200807 
15. Pandey P. K. A Consistent and Accurate Numerical Method for Approximate Numerical Solution of Two Point Boundary Value Problems. (To appear).

16. Jain M. K., Iyenger S. R. K., Jain R. K. Numerical Methods for Scientific and Engineering Computation (2/e). New Delhi, Willey Eastern Limited, 1987.

17. Gil M. I. Invertibility Conditions for Block Matrices and Estimates for Norms of Inverse Matrices. Rocky Mountain Journal of Mathematics, 2003, vol. 33, no. 4, pp. 1323-1335. DOI: $10.1216 / \mathrm{rmjm} / 1181075466$

18. Froberg C. E. Introduction to Numerical Analysis. New York, Addison-Wesley, 1969.

Pramod K. Pandey, PhD (Math), Associate Professor, Department of Mathematics, Dyal Singh College (University of Delhi) (Lodhi Road, New Delhi-110003, India), pramod_10p@hotmail.com.

\title{
ПАРНЫЙ ОБЪЕДИНЕННЫЙ РАЗНОСТНЫЙ МЕТОД ЧИСЛЕННОГО РЕШЕНИЯ НАЧАЛЬНО-КРАЕВЫХ ПАРАБОЛИЧЕСКИХ ЗАДАЧ ЧЕТВЕРТОГО ПОРЯДКА
}

\section{П. К. Панди}

\begin{abstract}
В данной статье мы предлагаем парный объединенный разностный метод численного решения параболических задач четвертого порядка. Для того, чтобы понизить сложность высшего порядка дифференциального члена, мы вводим промежуточную функцию, и, следовательно, краевая задача высокого порядка преобразуется в эквивалентную систему краевых задач более низкого порядка. Мы обсуждаем сходимость предложенного метода и проводим численные эксперименты для проверки и подтверждения эффективности и точности предложенного метода.

Ключевые слова: анализ сходимости; парный подход; параболическое уравнение четвертого порядка; началъная краевая задача; объединенный разностный метод.
\end{abstract}

\section{Литература}

1. Mullins, W. Theory of Thermal Grooving / W. Mullins // Journal of Applied Physics. - 1957. - V. 28, № 3. - P. 333-339.

2. Tritscher, P. An Integrable Fourth-Order Nonlinear Evolution Equation Applied to Surface Redistribution due to Capillarity / P. Tritscher // Journal of the Australian Mathematical Society, Ser. B. - 1997. - V. 38, № 4. - P. 518-541.

3. Myers, T. G. Thin Films with High Surface Tension / T. G. Myers // SIAM Review. 1998. - V. 40, № 3. - P. 441-462.

4. Kraus, H. Thin Elastic Shells / H. Kraus. - New York: John Wiley \& Sons Inc, 1967.

5. Schwartz, L. W. Theoretical and Numerical Results for Spin Coating of Viscous Liquids / L. W. Schwartz, R. V. Roy // Physics of Fluids. - 2004. - V. 16, № 3. P. 569-584. 
6. Allen, S. M. A Microscopic Theory for Antiphase Boundary Motion and its Application to Antiphase Domain Coarsening / S. M. Allen, J. W. Cahn // Acta Metall. - 1979. V. 27. - P. 1085-1095.

7. Bernoff, A. J. Singularities in a Modified Kuramoto - Sivashinsky Equation Describing Interface Motion for Phase Transition / A. J. Bernoff, A. L. Bertozzi // Physica D. 1995. - V. 85. - P. 375-404.

8. Rubinstein, J. Fast Reaction, Slow Diffusion and Curve Shortening / J. Rubinstein, P. Sternberg, J. B. Keller // SIAM Journal on Applied Mathematics. - 1989. - V. 49, № 1. - P. 116-133.

9. Khaliqa, A. Q. M. A Family of Second Order Methods for Variable Coefficient Fourth Order Parabolic Partial Differential Equations / A. Q. M. Khaliqa, E. H. Twizell // International Journal of Computer Mathematics. - 1987. - V. 23, № 1. - P. 63-76.

10. Mohanty, R. K. The Numerical Solution of Fourth Order Mildly Quasi-Linear Parabolic Initial Boundary Value Problem of Second Kind / R. K. Mohanty, D. J. Evans // International Journal of Computer Mathematics. - 2003. - V. 80, № 9. - P. 1147-1159.

11. Caglar, H. Fifth-Degree B-spline Solution for a Fourth-Order Parabolic Partial Differential Equations / H. Caglar, N. Caglar // Applied Mathematics and Computation. - 2008. - V. 201, № 1. - P. 597-603.

12. Soltanalizadeh, B. Application of Differential Transformation Method for Solving a Fourth-Order Parabolic Partial Differential Equations / B. Soltanalizadeh // International Journal of Pure and Applied Mathematics. - 2012. - V. 78, № 3. P. 299-308.

13. Dehghan, M. The Solution of the Variable Coefficients Fourth-Order Parabolic Partial Differential Equations by the Homotopy Perturbation Method / M. Dehghan, J. Manafian // Verlag der Zeitschrift fur Naturforschung, TuBbingen. - 2009. - V. 64.P. $420-430$.

14. Lick, W. A Consistent and Accurate Procedure for Obtaining Difference Equations from Differential Equation / W. Lick, T. Gaskins // International Journal For Numerical Methods In Engineering. - 1984. - V. 20. - P. 1433-1441.

15. Pandey, P. K. A Consistent and Accurate Numerical Method for Approximate Numerical Solution of Two Point Boundary Value Problems. (To appear).

16. Jain, M. K. Numerical Methods for Scientific and Engineering Computation (2/e) / M. K. Jain, S. R. K. Iyenger, R. K. Jain. - New Delhi: Willey Eastern Limited, 1987.

17. Gil, M. I. Invertibility Conditions for Block Matrices and Estimates for Norms of Inverse Matrices / M. I. Gil // Rocky Mountain Journal of Mathematics. - 2003. V. 33, №. 4. - P. 1323-1335.

18. Froberg, C. E. Introduction to Numerical Analysis / C. E. Froberg. - New York: Addison-Wesley, 1969.

Панди Прамод Кумар, кандидат физико-математических наук, доцент кафедрьи математики Колледжа Дъяла Сингха (Университет Дели) (Лодхи-Роуд, НъюДели-110003, Индия) pramod_10p@hotmail.com.

Поступила в редакиию 2 сентября 2020 г. 